\title{
Development board design of open electronics experimental system based on single chip microcomputer
}

\author{
Xingshan $\mathrm{Li}^{1, \mathrm{a}, *}$, Yuanshen GUO ${ }^{2}$, Jian $\mathrm{LU}^{3}$ \\ $1, *$ Luohe Medical College, Luohe, 462000, China \\ ${ }^{2}$ Henan Quality Engineering Vocational College, Pingdingshan, 467000, China \\ ${ }^{3}$ Henan Industrial Vocational and Technical College, Nanyang, 473000, China \\ aemail:604141388@qq.com
}

Keywords: SCM, FX-A full development board, power-supply module, SCM minimum system, Water lamp

\begin{abstract}
The study included for the innovative design of the image electronics course teaching experiment device, object oriented higher vocational college, school teachers and students. The results of the project to take a most intuitive and the most feasible way to replace the existing teaching equipment. It can be used at any time, and can meet the requirements of the students autonomous experiment after class.
\end{abstract}

\section{Introduction}

At present, most of the experimental equipment used in the teaching of image electronics courses are mainly two categories, One kind is the national each big teaching instrument company complete set of experiment table test equipment. Its large size, high cost, there are various types of instruments on the experimental stage, the experimental circuit is generally fixed, through the connection with a dedicated wire. Disadvantage is that the circuit can not be seen on the back of the device, easy to make students and beginners to understand the misunderstanding. The second category is to buy their own small desktop experimental device, the cost is low, but only a list of various types of experimental project process, there is no system of teaching methods to support. The significance and research value of this project lies in the knowledge points which are used in the electronic technology courses can be integrated with experimental project, for design an open experimental device with the improved teaching methods to achieve the best teaching effect. The package mainly includes five parts, respectively is FX-A full development board, programming adapte, FX-pro programming software, lucky star programmer and Fuxing function module.

\section{Functional of FX-A full development board}

FX-A full development board is composed of two major parts, which are software experimentation area and hardware experimentation area, cooperate With a variety of functional Modules and components to quickly develop a variety of applications. Experimental development board as shown in Figure 1 below.

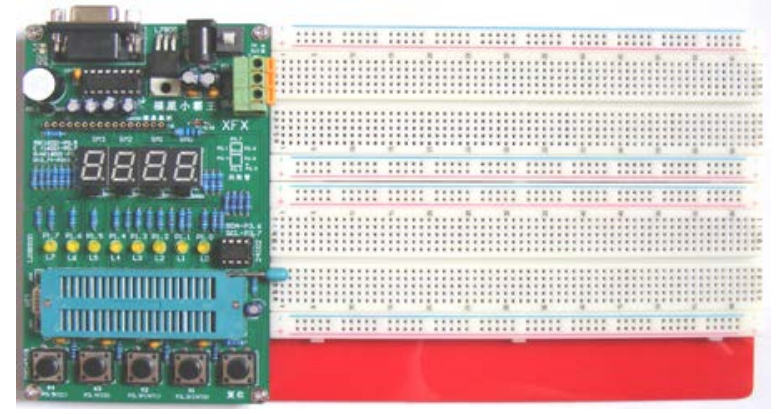

Fig. 1 FX-A full development board 
Development board adopt AT89S51 and AT89S52 as chip for experiment development. Two digital tube, eight light-emitting diodes (leds), four push button switch, a simple sound circuit, RS232 serial communication interface, serial memory, LCD display interface, etc were installed on the plate, as shown in figure 2.This experiment board not only can detect water lights, buzzer, LED dynamic scanning, the interrupt, counters, such as basic programming practice, but also can learn the I2C serial communication interface with PC, LCD display, etc. At present more popular technology[1].

At the same time, more convenient for beginners to understand the controlled object, in the experimental plate screen printing are marked on the surface of each control point of pin symbol, user-friendly rapid development on the experiment board control program.

\section{Performance of FX-A software experimentation area}

Development board software experimentation area is based on the students to learn microcontroller technology experimental requirements to develop a multi-function circuit board, able to complete as many as more than ten kinds of experiments and portfolio projects. And have ISP online programming function, as shown in figure 2. The components of the circuit board are described below[2].

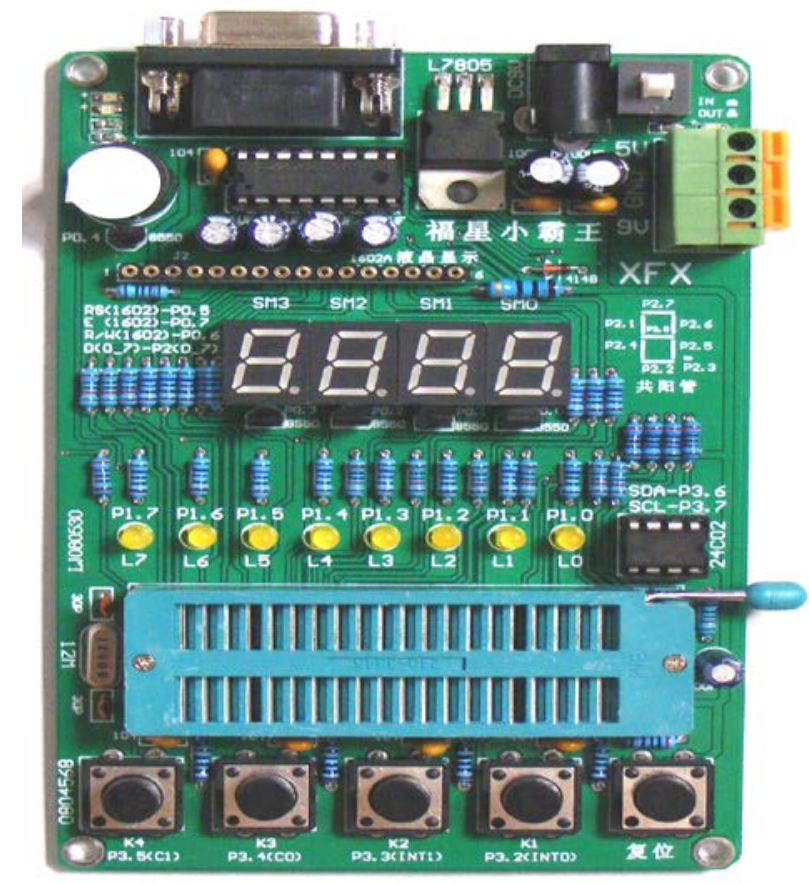

Fig.2 FX-A software experimental area

\section{Design of power supply module.}

Software experimentation area by the DC regulated power supply 9V input, after the 78L05 regulator, the output can be stabilized $5 \mathrm{~V}, 9 \mathrm{~V}$ DC power supply, the power supply requirements of the hardware experimentation area.The power switch of power switch only responsible for the board, which not only saves energy, also avoid the effect of receiving software experimental area in hardware experimental area of hardware connect circuit board[3]. As shown in figure 3. 




Fig. 3 Power supply module

\section{Design of single chip microcomputer minimum system.}

The MCU clock, reset circuit, clock oscillator circuit using $12 \mathrm{MHz}$,capacitor using 30pF,as shown in figure 4.RESET circuit adopts the button RESET circuit, the circuit besides have electricity RESET function, if you want to RESET, simply press the RESET button, the VCC power supply after the resistors R1 and R2 partial pressure, produce a RESET on the RESET end high level.

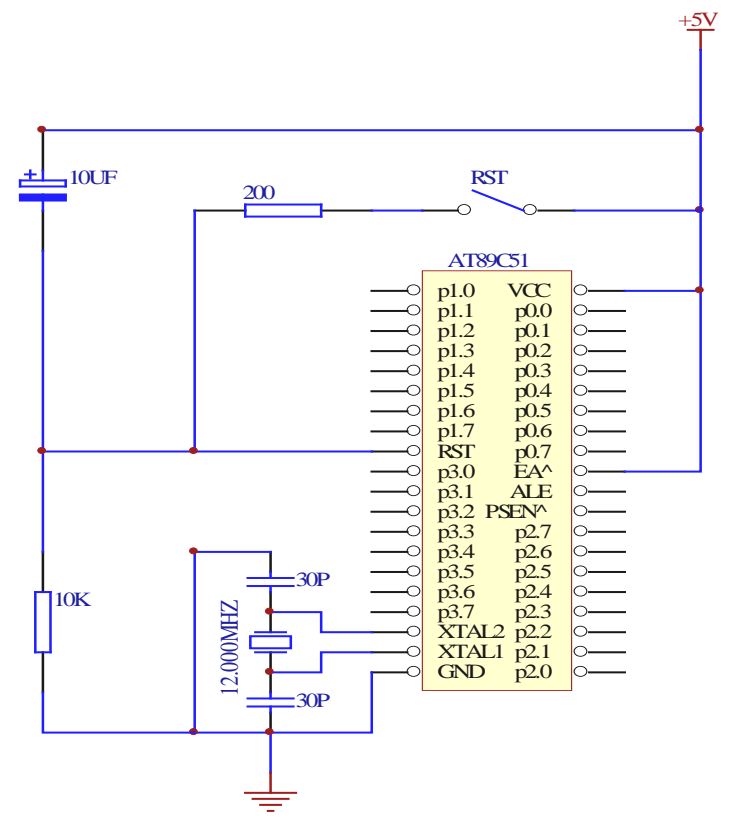

Fig.4 Single chip microcomputer minimum system

\section{Running water light display module.}

The P1 port of the main chip is connected with eight light emitting diodes, all of which are low level, that is, if the output of the P1 port is 0 , the corresponding lamp is bright, and if the output is 1 , the corresponding lamp is not bright, As shown in figure 5. 


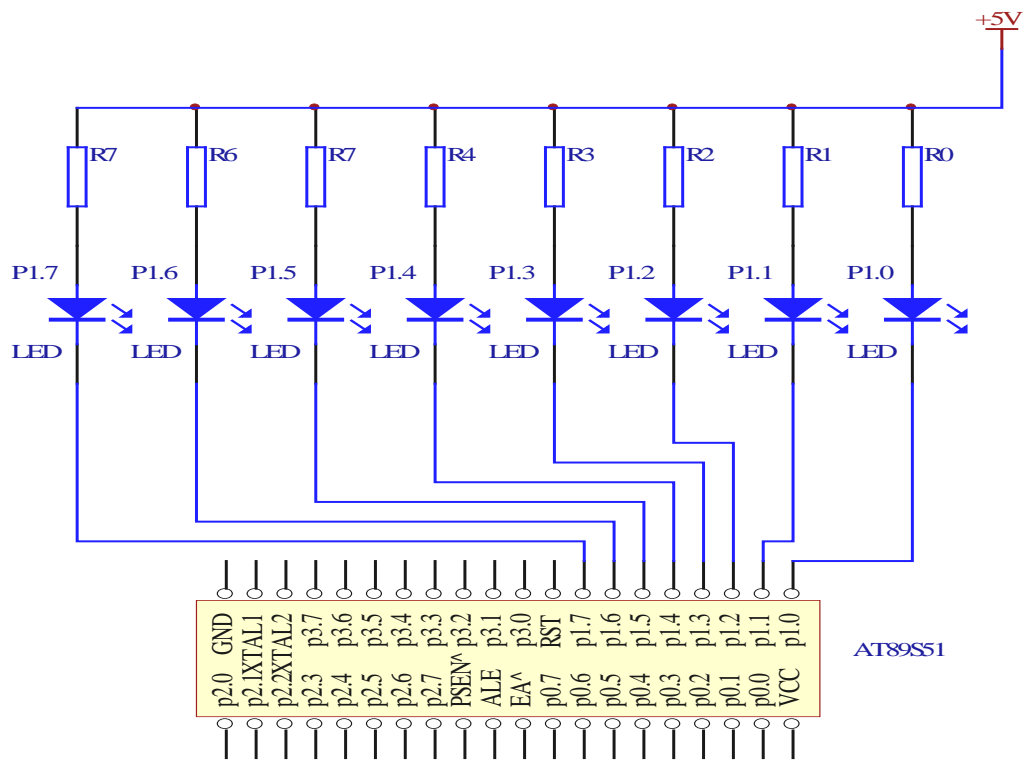

Fig.5 Running water light display module

\section{Dynamic display of digital tube.}

As shown in figure 6,main chip P0 and P2 port form the four digital tube driver circuit, low level effectively.Using the common anode LED digital tube, seven strokes external current limiting resistor and P2 each port is connected to the, use the PNP type triode tube is the common end (chip select) end of the drive, Emission Triode very even together, received is power supply terminal, base are respectively connected to p0.0 -- digital tube common sections of the chip select control P0.3. So, p0.0 - P0.3 in a pin output is low electricity at ordinary times, triode, power supply to the corresponding digital pipe, the bit digital tube is lit, the light pen, depending on these pen pin is high or low level. On the other hand, if a particular output pin is high level, transistor non conducting and can not give a corresponding digital tube power supply, the digital tube all written down is not bright[4].

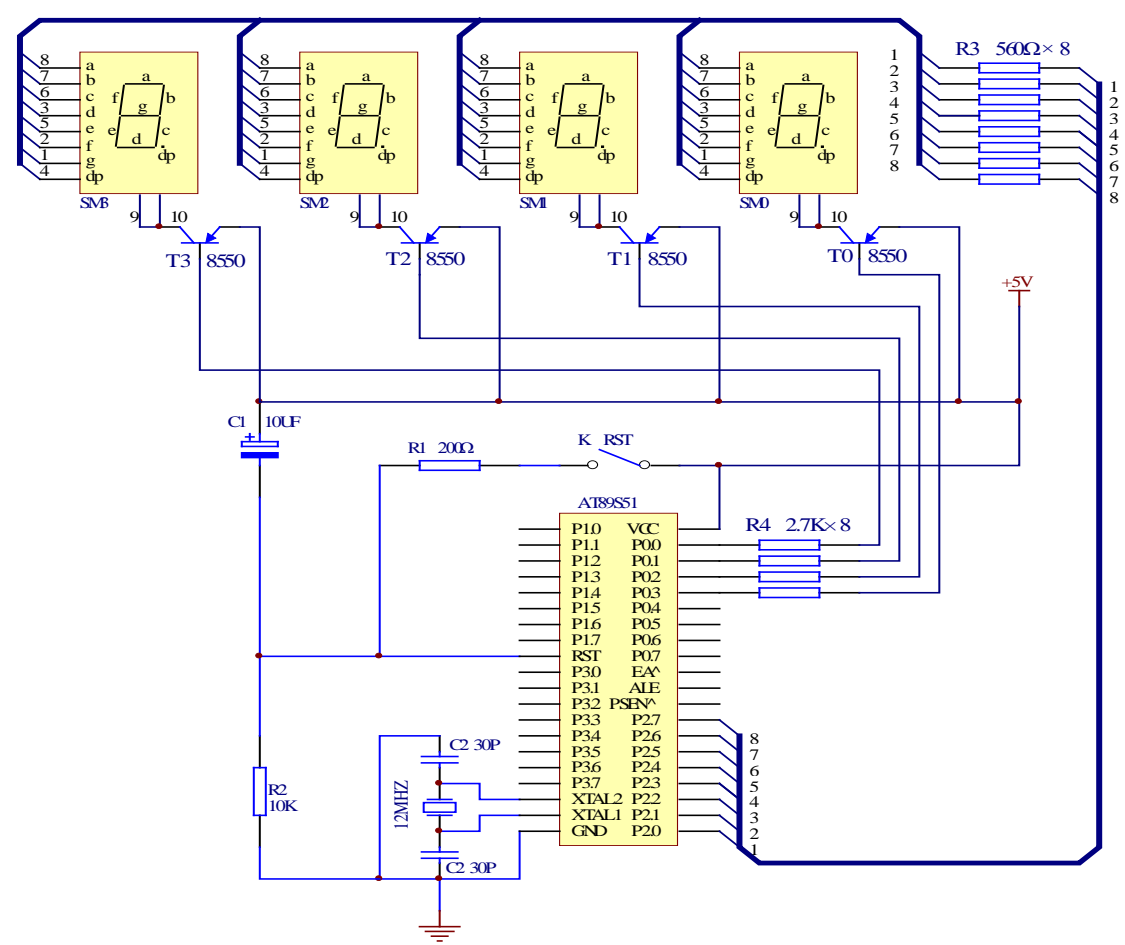

Fig.6 Digital tube dynamic display 


\section{Serial interface.}

Serial communication function is often used in the application of single chip microcomputer, is the only channel experiment board and PC communication, need to debug the program through serial interface to download to experiment board, the running state of the board program and partial results are to be uploaded to the PC through serial interface. As shown in figure 7.

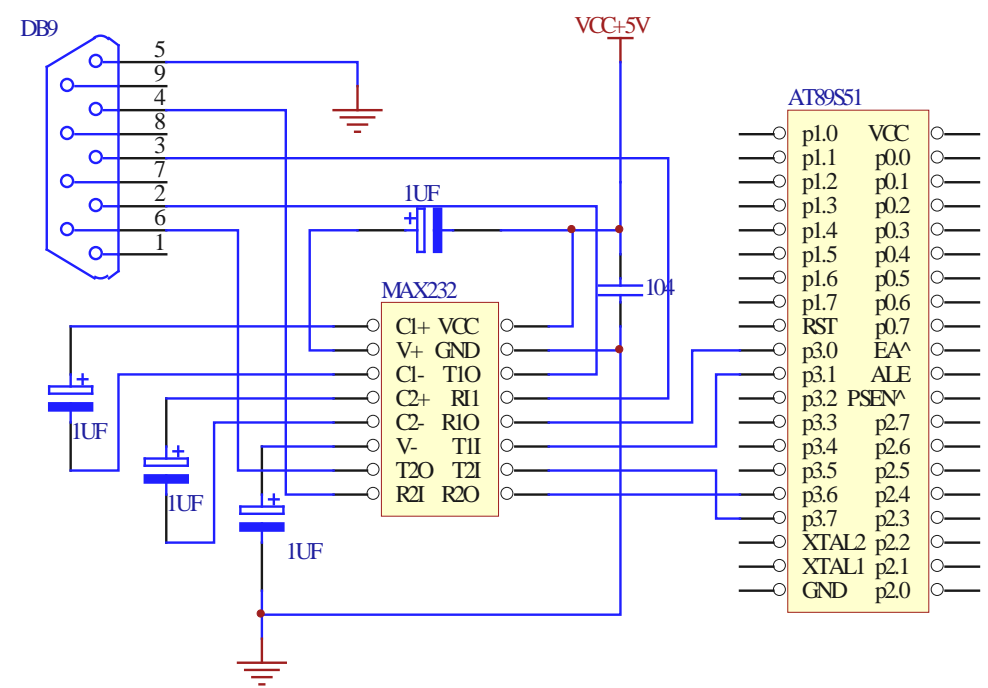

Fig. 7 MAX232 serial port communication module

PC serial interface RS - 232, RS - 232 includes electric of a bitwise serial transmission and regulations of mechanical aspects. RS - 232 requirement about electric property, drives the output voltage is relative to the signal ground between $5 \mathrm{v}$ to $15 \mathrm{v}$ for logic 1 level, said mark state; Relative to the output voltage signal ground between $+5 \mathrm{v} \sim+15 \mathrm{v}$ for logic 0 level, said the empty state. At the receiving end, logic level 1 to $3 \mathrm{v}$ to $15 \mathrm{v}$, logic 0 to $+3 \mathrm{v} \sim+15 \mathrm{v}$, which allows the sender to the receiver has $2 \mathrm{v}$ voltage drop. The RS - 232 level and TTL logic circuit (single-chip microcomputer) level is different, therefore, between PC and single chip microcomputer 89 s51 must pass a certain circuit switching logic level[5].

In direct communication with PC and single chip microcomputer, a selection of PC side 9-pin serial interface, so the RS - 232 only just need to a few lines to work normally. TXD/RXD are a pair of cable ,TXD for sending data output, RXD for receiving data input, when PC and single chip microcomputer in full-duplex mode directly communication, the two wires should be cross connection on both sides. There are more than these three lines (TXD, RXD and signal ground), single-chip microcomputer and PC can be respectively for asynchronous communication circuit chip programming, set to do not need any contact or handshake signal, direct way for data exchange.

The second function of the main chip of the P3 port pins P3.0, P3.1 is serial port RXD and TXD, its internal serial interface circuit with asynchronous communication function. Use MAX232 chip, MCU TTL level conversion required by RS232 level and RS232 level translation for TTL level, there have a charge pump inside the chip , as long as the single $5 \mathrm{~V}$ power supply can be generated by high voltage, the use is very convenient, chip peripheral circuit is simple, four 1 micro method of capacitors can be.

Key input.

Main chip P3.2 - P3.5 took four push button switch, the experimental plate in order to consider the anti-jamming, the input pin and appropriate pull-up resistor ,as shown in figure 7. 


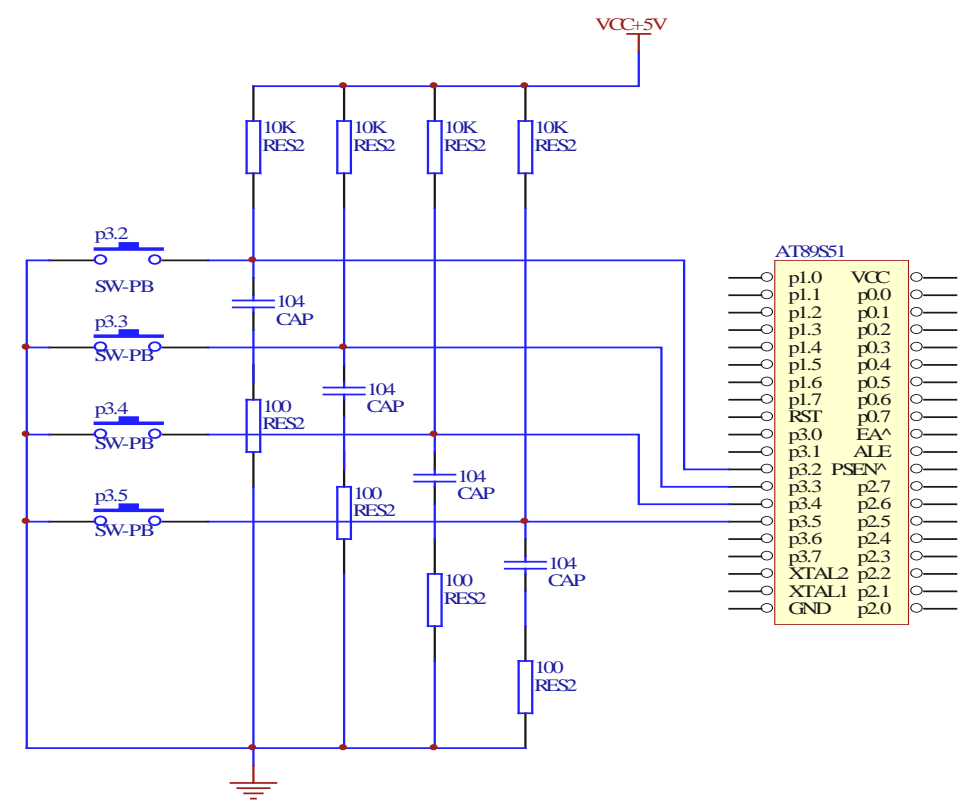

Fig. 8 The key input module

\section{Summary}

After test, all test parameters of the circuit board has reached the design requirements.

\section{Acknowledgement}

In this paper, the research was sponsored by the education technology and equipment and practice education research project topics in Henan Province In 2015 (Project No. GZS112).

\section{References}

[1] Duan Guo-yan. Electronic technology of virtual experiment system research and development[D]. Southwest Jiaotong University, Chongqing, 2013

[2] Xinyu Zhu. The development and application of electronic design automation experiment system[D]. Beijing University of Posts and Telecommunications, Beijing, 2012

[3] Chunyu Cheng and so on. The development of the analog electronic technology is suitable for hierarchical teaching experiment platform [J]. Laboratory Science, 2014(5)

[4] Shisheng Yan and so on. The development of the new type of analog electronic technology experiment device[J]. Journal of hainan normal university (natural science edition), 2012(3)

[5] Su Lei. The development and application of long electrician laboratory equipment[J]. Electronic Design Engineering ,2011(9) 